\title{
A Teleoperation Interface for Loco-Manipulation Control of Mobile Collaborative Robotic Assistant
}

\author{
Yuqiang Wu ${ }^{\circledR}$, Pietro Balatti ${ }^{\circledR}$, Marta Lorenzini ${ }^{\circledR}$, Fei Zhao, Wansoo Kim ${ }^{\circledR}$, and Arash Ajoudani (i)
}

\begin{abstract}
This letter presents a novel teleoperation interface that enables remote loco-manipulation control of a MObile Collaborative robotic Assistant (MOCA). MOCA is a new research platform developed at the Istituto Italiano di Tecnologia (IIT), which is composed of a lightweight manipulator arm, a Pisa/IIT SoftHand, and a mobile platform driven by four omni-directional wheels. A whole-body impedance controller is consequently developed to ensure accurate tracking of the impedance and position trajectories at MOCA end-effector by considering the causal interactions in such a dynamic system. The proposed teleoperation interface provides the user with two control modes: locomotion and manipulation. The locomotion mode receives inputs from a personalized human center-of-pressure model, which enables real-time navigation of the MOCA mobile base in the environment. The manipulation mode receives inputs from a tele-impedance interface, which tracks human arm endpoint stiffness and trajectory profiles in real time and replicates them using the MOCA's whole-body impedance controller. To evaluate the performance of the proposed teleoperation interface in the execution of remote tasks with dynamic uncertainties, a sequence of challenging actions, i.e., navigation, door opening, and wall drilling, has been considered in the experimental setup.
\end{abstract}

Index Terms-Telerobotics and teleoperation, physical humanrobot interaction, mobile manipulation.

Manuscript received February 23, 2019; accepted June 28, 2019. Date of publication July 15, 2019; date of current version July 24, 2019. This letter was recommended for publication by Associate Editor K. Hambuchen and Editor A. M. Okamura upon evaluation of the reviewers' comments. This work was carried out at the $\mathrm{HRI}^{2} \mathrm{Lab}$, Istituto Italiano di Tecnologia, Genoa, Italy. In this work, Y. Wu was also supported by the China Scholarship Council. (Yuqiang Wu and Pietro Balatti contributed equally to this work.) (Corresponding author: Pietro Balatti.)

$\mathrm{Y}$. Wu is with the State Key Laboratory for Manufacturing System Engineering, the Key Laboratory of Intelligent Robots, and the School of Mechanical Engineering, Xi' an Jiaotong University, Xi' an 710049, China, and also with the $\mathrm{HRI}^{2} \mathrm{Lab}$, Istituto Italiano di Tecnologia, 16163 Genoa, Italy (e-mail: wuyuqiang@stu.xjtu.edu.cn).

P. Balatti is with the $\mathrm{HRI}^{2}$ Lab, Istituto Italiano di Tecnologia, 16163 Genoa, Italy, and also with the Department of Information Engineering, University of Pisa, 56126 Pisa, Italy (e-mail: pietro.balatti@iit.it).

M. Lorenzini is with the $\mathrm{HRI}^{2}$ Lab, Istituto Italiano di Tecnologia, 16163 Genoa, Italy, and also with the Department of Electronics, Information and Bioengineering, Politecnico di Milano, 20133 Milano, Italy (e-mail: marta.lorenzini@iit.it).

F. Zhao is with the State Key Laboratory for Manufacturing System Engineering, the Key Laboratory of Intelligent Robots, and the School of Mechanical Engineering, Xi' an Jiaotong University, Xi' an 710049, China (e-mail: ztzhao@mail.xjtu.edu.cn).

W. Kim and A. Ajoudani are with the $\mathrm{HRI}^{2} \mathrm{Lab}$, Istituto Italiano di Tecnologia, 16163 Genoa, Italy (e-mail: Wan-Soo.Kim@iit.it; arash.ajoudani@iit.it).

This letter has supplementary downloadable material available at http:// ieeexplore.ieee.org, provided by the authors.

Digital Object Identifier 10.1109/LRA.2019.2928757

\section{INTRODUCTION}

$\mathbf{T}$ ELEOPERATION technologies are widely used in space exploration [1], disaster relief [2], surgery [3] and surveillance due to the existence of risks to humans or unreachable physical distances. A typical teleoperation system is usually constructed in a master-slave architecture, in which the key components are control methods developed for slave robot, humanrobot interfaces sending commands to robot while receiving a feedback for human, and communication support to avoid large time delays [4]. Position- or velocity-based teleoperation systems with force feedback are among the most common to benefit from such an architecture [5].

More recently, the introduction of active impedance control techniques in the teleoperation control architecture has made significant improvements in robot responses to the uncertainties arising from the remote task dynamics or the environment [6] In most cases, however, the impedance parameters in teleoperation systems are kept constant, which limits the interaction performance of robots in the execution of highly dynamic tasks such as drilling, door opening, valve turning, chipping, and may result in task failure.

Towards delivering a more intuitive and effective way to adjust the impedance parameters of teleoperated robots in such interaction scenarios, Ajoudani, et al., proposed the concept of tele-impedance [7], as an alternative technique to unilateral and bilateral teleoperation. Tele-impedance enriches the command sent to the slave robot by combing the master's estimated position and the stiffness references. The compound reference commands are then realized by the remote impedance controller without explicit force feedback to the operator. The application of tele-impedance in remote control of robotic arm [8], hand [9], and dual-arm [10] systems has demonstrated this control concept's high potential. Nevertheless, tele-impedance control method has only been employed in the teleoperation of fixedbase platforms.

On the other hand, modern teleoperation systems call for the crucial contribution of mobility to enable their users to navigate the systems to the points of interest. Additionally, the mobile base extends the workspace of the manipulator and provides improved flexibility brought by the extra degrees of freedom (DoF). However, although a wide variety of mobile robots with legged (e.g., WALK-MAN [11], TORO [12]) or wheeled (e.g., Justin [13], TWENDY-ONE [14]) locomotion capabilities exist, the lack for an intuitive and interaction-efficient interface for the control of robot loco-manipulation has prohibited their deployment in challenging remote interaction scenarios. In fact, most of the current mobility based teleoperation interfaces (e.g. [15], 

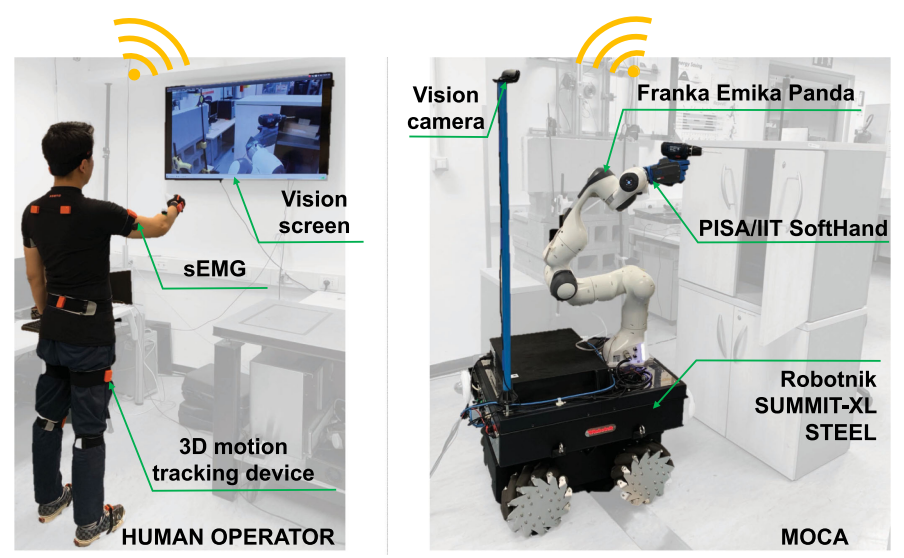

Fig. 1. The human operator remotely teleoperates MOCA by moving his/her body, thanks to the visual feedback shown on the screen and provided by the camera mounted on the robot.

[16]) focus on the mobility aspect and do not provide effective solutions for the control of remote interactions in challenging manipulation tasks.

Although mobility brings more flexibility, it also increases the difficulty to design a friendly loco-manipulation interface for the operator to control a mobile manipulator. In most cases, a joystick interface is used due to its simplicity [17]. However, when dealing with many DoF, it could be confusing for the users. Graphic user interface (GUI) running on a computer is also widely adopted in the teleoperation of mobile manipulators [18], although a complex GUI may be not intuitive and effective enough for the operators. Haptic devices such as Falcon are also used in some cases to get force feedback [19], although most of them have only six DoF and are designed to control a fixed manipulator, not a mobile one. In a few situations [20], [21], an exoskeleton or an extra manipulator is employed to measure the position of human arm and provide force feedback to human, which makes teleoperation more intuitive. However, these interfaces increase the system complexity.

Accordingly, the aim of this letter is to propose a novel teleoperation interface for control of robot loco-manipulation in remote environments. We first built a new MObile Collaborative robotic Assistant (MOCA), as shown in Fig. 1. Next, a wholebody impedance controller is implemented for MOCA to enable accurate tracking of its end-effector position and impedance profiles by considering the causal interactions in such a dynamic chain. Furthermore, to merge the tele-impedance control with robot locomotion ability, an advanced teleoperation interface is presented to enable MOCA control in two modes: Locomotion and Manipulation. To validate the proposed interface in enabling agile locomotion and manipulation control of MOCA in remote environments, experiments including navigation, door opening and wall drilling, have been carried out.

The remainder of this letter is structured as follows: Section II introduces the system hardware integration, and details of whole-body dynamics and impedance controller design. The teleoperation interface is presented in Section III. Section IV provides details of the task-based control flow. Experimental setup and the results are introduced in Sections V and VII, respectively.

\section{MObile Collaborative Robotic Assistant (MOCA)}

The overall control architecture can be divided into two main components as shown in Fig. 2: MOCA and the human operator. This section provides details of the MOCA system integration, and the development of its whole-body impedance controller. MOCA is designed as a versatile platform for advancing research on human-robot interaction and collaboration, with potential applications in flexible manufacturing and teleoperation scenarios. The input to this controller is given by the human-robot loco-manipulation interface that will be presented in Section III.

\section{A. System Hardware Integration}

MOCA is the result of the integration of four components, as shown in Fig. 1: Franka Emika Panda robotic arm, equipped with the underactuated Pisa/IIT SoftHand, which are mounted on top of Robotnik SUMMIT-XL STEEL mobile platform. A commercial camera supported by a pole is also added to the mobile base.

Franka Emika Panda is a torque-controlled lightweight robot arm. It has seven Degrees of Freedom (DoF) which provide more flexibility in motion. Each joint is equipped with a torque sensor on the link side, achieving a better control performance in presence of model uncertainties.

Robotnik SUMMIT-XL STEEL is driven by four Omnidirectional wheels, that allows the platform to avoid nonholonomic constraints. The Cartesian velocity control interface is offered with a high gain in low level, which implies that the dynamics of the mobile platform can be omitted. It provides the odometry data, computed by Extended Kalman Filter (EKF) with the use of a high precision inertial measurement unit and the wheels' velocity.

The idea of adaptive synergies [22] which comes from the combination of natural motor control principles is the core of the Pisa/IIT SoftHand. As a result the hand can adapt itself according to the physical interaction of its body with the object, allowing to grasp a wide range of objects despite its single degree of actuation. The features of simplicity, lightness, robustness and compliance make it an ideal choice for integration to MOCA robot.

Although each of the above components possesses particular advantages, when integrated in one system, they present significant challenges. First of all, the control interface of Franka Emika Panda, Robotnik SUMMIT-XL STEEL and Pisa/IIT SoftHand are respectively torque-based, velocity-based, and current-based (underactuated). Hence, the causal interactions in such a dynamical system must be considered in control of robot interaction controller [23]. Secondly, this integration introduces more redundancy (the manipulator and the mobile platform have 7-DoF and 3-DoF, respectively), which adds complexity to the control. Furthermore, the robotic arm and the mobile platform have different bandwidths: the mobile platform typically has a slower dynamic response than the manipulator [24], which must be taken into account.

\section{B. Whole-Body Dynamics Analysis}

First, we consider the dynamic models of the manipulator and of the mobile platform separately, each one defined in its own 


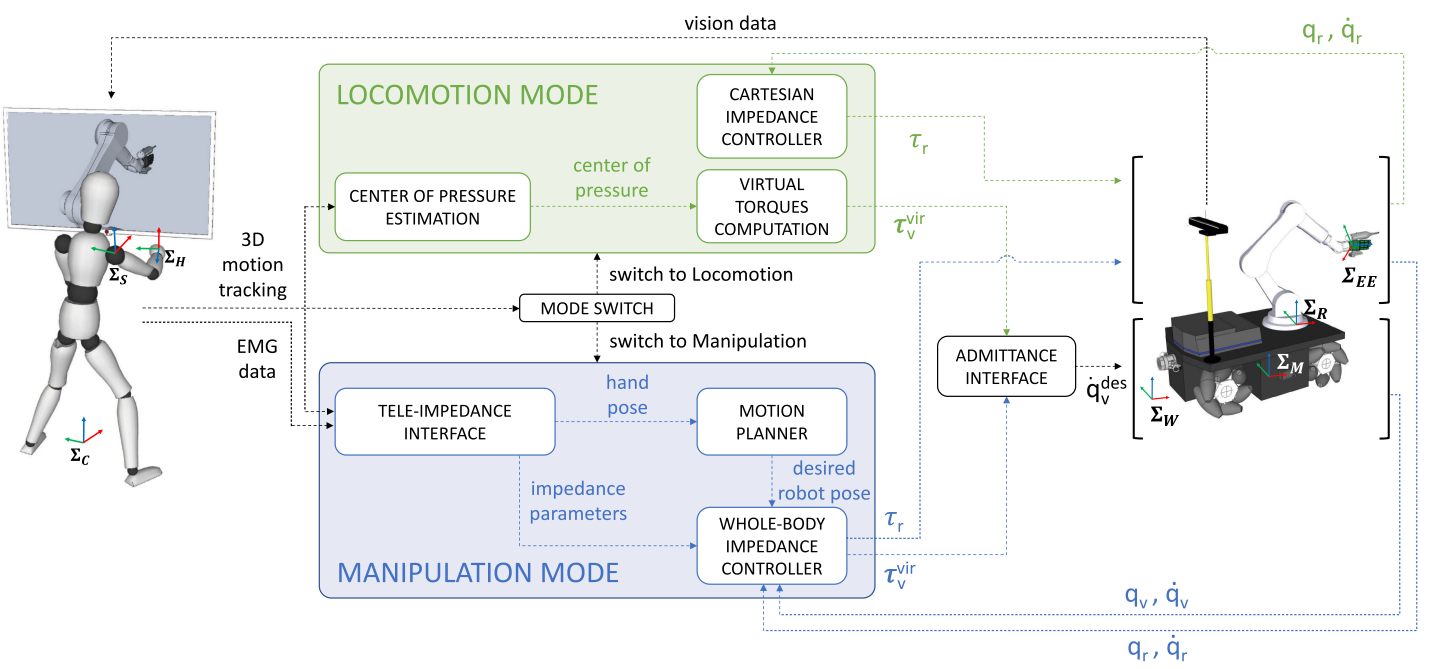

Fig. 2. The control architecture of the presented framework. The dotted lines represent the data exchanged by the modules: in green the data exchanged during Locomotion, in blue during Manipulation, and in black the data that always flow when the system is active.

base frame. The reference frames are represented in the right side of Fig. 2: world frame $\Sigma_{W}$, mobile platform base frame $\Sigma_{M}$, and manipulator base frame $\Sigma_{R}$. As it is well known, a robotic manipulator is a coupled, time-varying and nonlinear system. The general dynamic model of a manipulator with joint coordinates $\boldsymbol{q}_{r} \in \mathbb{R}^{n}$ can be written as

$$
\boldsymbol{M}_{r}\left(\boldsymbol{q}_{r}\right) \ddot{\boldsymbol{q}}_{r}+\boldsymbol{C}_{r}\left(\boldsymbol{q}_{r}, \dot{\boldsymbol{q}}_{r}\right)+\boldsymbol{g}_{r}\left(\boldsymbol{q}_{r}\right)=\boldsymbol{\tau}_{r}+\boldsymbol{\tau}_{r}^{\text {ext }},
$$

where $\boldsymbol{M}_{r} \in \mathbb{R}^{n \times n}$ is the symmetric and positive definite inertial matrix of the arm. $\boldsymbol{C}_{r} \in \mathbb{R}^{n}$ is the Coriolis and centrifugal force, $\boldsymbol{g}_{r} \in \mathbb{R}^{n}$ is the gravity vector, $\boldsymbol{\tau}_{r} \in \mathbb{R}^{n}$ and $\boldsymbol{\tau}_{r}^{\text {ext }} \in \mathbb{R}^{n}$ are the commanded torque vector and external torque vector, respectively. In (1), it is maintained the property that $\dot{\boldsymbol{M}}_{r}-2 \boldsymbol{C}_{r} \in \mathbb{R}^{n \times n}$ is skew symmetric.

The dynamics of a mobile platform with $m$ DoF, $\boldsymbol{q}_{v} \in \mathbb{R}^{m}$, can be obtained using the Lagrangian approach in the following form [25]:

$$
\boldsymbol{M}_{v}\left(\boldsymbol{q}_{v}\right) \ddot{\boldsymbol{q}}_{v}+\boldsymbol{C}_{v}\left(\boldsymbol{q}_{v}, \dot{\boldsymbol{q}}_{v}\right)=\boldsymbol{E}_{v}\left(\boldsymbol{q}_{v}\right) \boldsymbol{\tau}_{v}-\boldsymbol{A}_{v}^{T}\left(\boldsymbol{q}_{v}\right) \lambda,
$$

where $\boldsymbol{M}_{v} \in \mathbb{R}^{m \times m}$ is the symmetric and positive definite inertial matrix of the mobile platform, $\boldsymbol{C}_{v} \in \mathbb{R}^{m}$ is the centrifugal force, $\boldsymbol{E}_{v} \in \mathbb{R}^{m \times m}$ is the input transformation matrix, $\boldsymbol{\tau}_{v} \in \mathbb{R}^{m}$ is the commanded torque vector, $\boldsymbol{A}_{v} \in \mathbb{R}^{m \times m}$ is the constraint matrix and $\lambda \in \mathbb{R}^{m}$ is the Lagrange multiplier which denotes the constraint force vector.

When integrating the manipulator and the mobile platform into one mobile manipulation system, there exists a dynamic interaction between these two subsystems. The new dynamic equations of the manipulator and the mobile platform subject to each other are given by (3) and (4) [24]:

$$
\begin{aligned}
& \boldsymbol{M}_{r}\left(\boldsymbol{q}_{r}\right) \ddot{\boldsymbol{q}}_{r}+\boldsymbol{C}_{r}\left(\boldsymbol{q}_{r}, \dot{\boldsymbol{q}}_{r}\right)+\boldsymbol{C}_{r v}\left(\boldsymbol{q}_{r}, \dot{\boldsymbol{q}}_{r}, \dot{\boldsymbol{q}}_{\boldsymbol{v}}\right)+\boldsymbol{g}_{r}\left(\boldsymbol{q}_{r}\right) \\
& \quad=\boldsymbol{\tau}_{r}+\boldsymbol{\tau}_{r}^{e x t}-\boldsymbol{R}_{r}\left(\boldsymbol{q}_{r}, \boldsymbol{q}_{v}\right) \ddot{\boldsymbol{q}}_{v} .
\end{aligned}
$$

where $\boldsymbol{C}_{r v} \in \mathbb{R}^{n}$ represents Coriolis and centrifugal terms caused by angular motion of the mobile platform, and $\boldsymbol{R}_{r} \in$ $\mathbb{R}^{n \times m}$ is the inertial matrix which represents the effect of the mobile platform dynamics on the manipulator.

$$
\boldsymbol{M}_{v}\left(\boldsymbol{q}_{v}\right) \ddot{\boldsymbol{q}}_{v}+\boldsymbol{C}_{v}\left(\boldsymbol{q}_{v}, \dot{\boldsymbol{q}}_{v}\right)+\boldsymbol{C}_{v r}\left(\boldsymbol{q}_{v}, \boldsymbol{q}_{r}, \dot{\boldsymbol{q}}_{v}, \dot{\boldsymbol{q}}_{r}\right)
$$

$$
\begin{aligned}
= & \boldsymbol{E}_{v}\left(\boldsymbol{q}_{v}\right) \boldsymbol{\tau}_{v}-\boldsymbol{A}_{v}^{T}\left(\boldsymbol{q}_{v}\right) \lambda-\boldsymbol{M}_{v r}\left(\boldsymbol{q}_{v}, \boldsymbol{q}_{r}\right) \ddot{\boldsymbol{q}}_{v} \\
& -\boldsymbol{R}_{v}\left(\boldsymbol{q}_{v}, \boldsymbol{q}_{r}\right) \ddot{\boldsymbol{q}}_{r},
\end{aligned}
$$

where $\boldsymbol{M}_{v r} \in \mathbb{R}^{m \times m}$ and $\boldsymbol{C}_{v r} \in \mathbb{R}^{m}$ denote the inertial term and Coriolis and centrifugal terms due to the presence of the manipulator, $\boldsymbol{R}_{v} \in \mathbb{R}^{m \times n}$ is the inertial matrix which reflects the dynamic effect of the manipulator motion on the mobile platform.

As mentioned above, the mobile platform presents a velocitybased control and a high gain is set in the low level velocity controller. This means that the dynamics of the mobile platform can be omitted and any external dynamic effect from the manipulator can be ignored. Considering that we aim at achieving a whole-body impedance control law, a force-torque interface is preferred. As a result, we implemented a Cartesian admittance controller based on the velocity interface:

$$
\boldsymbol{M}_{a d m} \ddot{\boldsymbol{q}}_{v}^{d e s}+\boldsymbol{D}_{a d m} \dot{\boldsymbol{q}}_{v}^{d e s}=\boldsymbol{\tau}_{v}^{v i r}+\boldsymbol{\tau}_{v}^{e x t}
$$

where $\boldsymbol{M}_{a d m} \in \mathbb{R}^{m \times m}$ and $\boldsymbol{D}_{a d m} \in \mathbb{R}^{m \times m}$ are the virtual inertial and virtual damping, $\dot{\boldsymbol{q}}_{v}^{\text {des }} \in \mathbb{R}^{m}$ is the desired velocity sent to the mobile platform, $\boldsymbol{\tau}_{v}^{v i r} \in \mathbb{R}^{m}$ and $\boldsymbol{\tau}_{v}^{\text {ext }} \in \mathbb{R}^{m}$ are the virtual and external torque interfaces.

According to the tasks we plan to perform (introduced in Section V), in the Manipulation mode, the mobile platform can be considered in a quasi-static state. The mobile platform motion does not have much effect on the manipulator. Therefore, the dynamic coupling terms in (3) can be neglected. Consequently, the overall dynamics of MOCA can be formulated as

$$
\begin{gathered}
\left(\begin{array}{cc}
\boldsymbol{M}_{a d m} & \mathbf{0} \\
\mathbf{0} & \boldsymbol{M}_{r}
\end{array}\right)\left(\begin{array}{l}
\ddot{\boldsymbol{q}}_{v} \\
\ddot{\boldsymbol{q}}_{r}
\end{array}\right)+\left(\begin{array}{cc}
\boldsymbol{D}_{a d m} & \mathbf{0} \\
\mathbf{0} & \boldsymbol{C}_{r}
\end{array}\right)\left(\begin{array}{c}
\dot{\boldsymbol{q}}_{v} \\
\dot{\boldsymbol{q}}_{r}
\end{array}\right)+\left(\begin{array}{c}
\mathbf{0} \\
\boldsymbol{g}_{r}
\end{array}\right) \\
=\left(\begin{array}{c}
\boldsymbol{\tau}_{v}^{v i r} \\
\boldsymbol{\tau}_{r}
\end{array}\right)+\left(\begin{array}{c}
\boldsymbol{\tau}_{v}^{e x t} \\
\boldsymbol{\tau}_{r}^{e x t}
\end{array}\right) .
\end{gathered}
$$

So far, a simplified whole-body dynamic formula has been obtained. We can get the two subsystem decoupled from a dynamic point of view because of the following two assumptions. 
Assumption 1: The gain of the low level velocity controller is high enough to compensate any dynamic effects from the external.

Assumption 2: In the Manipulation mode, the mobile platform is in a quasi-static state. Hence, the mobile platform motion does not have much effect on the manipulator.

According to the above analysis, the assumptions are feasible in our situation. In the following, a whole-body impedance controller is established based on the decoupled dynamics.

\section{Whole-Body Impedance Controller Implementation}

For a manipulator with joint coordinates $\boldsymbol{q} \in \mathbb{R}^{n}$, the desired dynamic relationship between Cartesian error $\tilde{\boldsymbol{x}} \in \mathbb{R}^{6}$ and external force $\boldsymbol{F}_{\text {ext }} \in \mathbb{R}^{6}$ in the Cartesian impedance controller is given by [26]:

$$
\boldsymbol{\Lambda}(\boldsymbol{x}) \ddot{\tilde{\boldsymbol{x}}}+\left(\boldsymbol{\mu}(\boldsymbol{x}, \dot{\boldsymbol{x}})+\boldsymbol{D}_{d}\right) \dot{\tilde{\boldsymbol{x}}}+\boldsymbol{K}_{d} \tilde{\boldsymbol{x}}=\boldsymbol{F}_{\text {ext }},
$$

where $\boldsymbol{K}_{d} \in \mathbb{R}^{6 \times 6}$ and $\boldsymbol{D}_{d} \in \mathbb{R}^{6 \times 6}$ are the desired Cartesian stiffness and damping respectively. $\boldsymbol{\Lambda}(\boldsymbol{x}) \in \mathbb{R}^{6 \times 6}$ represents Cartesian inertial and $\boldsymbol{\mu}(\boldsymbol{x}, \dot{\boldsymbol{x}}) \in \mathbb{R}^{6 \times 6}$ represents Cartesian Coriolis and centrifugal matrix. They can be computed respectively by (8) and (9):

$$
\begin{aligned}
\boldsymbol{\Lambda}(\boldsymbol{x}) & =\boldsymbol{J}(\boldsymbol{q})^{-T} \boldsymbol{M}(\boldsymbol{q}) \boldsymbol{J}(\boldsymbol{q})^{-1} \\
\boldsymbol{\mu}(\boldsymbol{x}, \dot{\boldsymbol{x}}) & =\boldsymbol{J}(\boldsymbol{q})^{-T}\left(\boldsymbol{C}(\boldsymbol{q}, \dot{\boldsymbol{q}})-\boldsymbol{M}(\boldsymbol{q}) \boldsymbol{J}(\boldsymbol{q})^{-1} \dot{\boldsymbol{J}}(\boldsymbol{q})\right) \boldsymbol{J}(\boldsymbol{q})^{-1},
\end{aligned}
$$

where $\boldsymbol{M}(\boldsymbol{q}) \in \mathbb{R}^{n \times n}$ represents the inertial matrix, $\boldsymbol{C}(\boldsymbol{q}, \dot{\boldsymbol{q}}) \in$ $\mathbb{R}^{n \times n}$ is the Coriolis and centrifugal matrix and $\boldsymbol{J}(\boldsymbol{q}) \in \mathbb{R}^{6 \times n}$ represents the Jacobian matrix.

The Cartesian impedance controller input for the main task is as following:

$$
\begin{aligned}
\boldsymbol{\tau}_{i m p}= & \boldsymbol{g}(\boldsymbol{q})+\boldsymbol{J}(\boldsymbol{q})^{T}\left(\boldsymbol{\Lambda}(\boldsymbol{x}) \ddot{\boldsymbol{x}}_{d}+\boldsymbol{\mu}(\boldsymbol{x}, \dot{\boldsymbol{x}}) \dot{\boldsymbol{x}}_{\boldsymbol{d}}\right. \\
& \left.-\boldsymbol{K}_{d} \tilde{\boldsymbol{x}}-\boldsymbol{D}_{d} \dot{\tilde{\boldsymbol{x}}}\right),
\end{aligned}
$$

where $\boldsymbol{g}(\boldsymbol{q}) \in \mathbb{R}^{n}$ denotes the gravity force and $\boldsymbol{x}_{d} \in \mathbb{R}^{6}$ is the desired Cartesian position and orientation.

For redundant robot arm, the null-space task input is defined as:

$$
\boldsymbol{\tau}_{n u l l}=\boldsymbol{N}(\boldsymbol{q})\left(-\boldsymbol{D}_{n} \dot{\boldsymbol{q}}-\boldsymbol{K}_{n}\left(\boldsymbol{q}-\boldsymbol{q}_{d, 0}\right)\right),
$$

where $\boldsymbol{K}_{n} \in \mathbb{R}^{n \times n}$ and $\boldsymbol{D}_{n} \in \mathbb{R}^{n \times n}$ are the desired Cartesian stiffness and damping of the null-space task, $\boldsymbol{q}_{d, 0} \in \mathbb{R}^{n}$ is the virtual equilibrium position, $\boldsymbol{N}(\boldsymbol{q}) \in \mathbb{R}^{n \times n}$ is the projection matrix in order to prevent interference with the Cartesian impedance behavior. Here, the following dynamically consistent projection proposed by Khatib [27] is employed:

$$
\boldsymbol{N}(\boldsymbol{q})=\boldsymbol{I}-\boldsymbol{J}^{T}(\boldsymbol{q}) \boldsymbol{\Lambda}(\boldsymbol{q}) \boldsymbol{J}(\boldsymbol{q}) \boldsymbol{M}^{-1}(\boldsymbol{q}) .
$$

To implement whole-body Cartesian impedance control for MOCA, whole-body forward kinematics $\boldsymbol{x}_{w}(\boldsymbol{q}) \in \mathbb{R}^{6}$, wholebody Jacobian $\boldsymbol{J}_{w}(\boldsymbol{q}) \in \mathbb{R}^{6 \times 10}$, whole body Cartesian inertial $\boldsymbol{\Lambda}_{w}\left(\boldsymbol{x}_{w}\right) \in \mathbb{R}^{6 \times 6}$, Cartesian Coriolis/centrifugal $\boldsymbol{\mu}\left(\boldsymbol{x}_{w}, \dot{\boldsymbol{x}}_{\boldsymbol{w}}\right) \in$ $\mathbb{R}^{6 \times 6}$ and null-space projection matrix $N(\boldsymbol{q}) \in \mathbb{R}^{10 \times 10}$ have to be constructed.

The forward kinematics transform of MOCA at the endeffector frame $\Sigma_{E E}$ with respect to the world frame $\Sigma_{W}$, $\boldsymbol{T}_{E E}^{W}(\boldsymbol{q}) \in \mathbb{R}^{4 \times 4}$ can be derived as follows:

$$
\boldsymbol{T}_{E E}^{W}(\boldsymbol{q})=\boldsymbol{T}_{M}^{W}\left(\boldsymbol{q}_{v}\right) \boldsymbol{T}_{R}^{M} \boldsymbol{T}_{E E}^{R}\left(\boldsymbol{q}_{r}\right)
$$

where $\boldsymbol{q}=\left(\boldsymbol{q}_{v}, \boldsymbol{q}_{r}\right)^{T} \in \mathbb{R}^{10}$ represents whole-body joint variables, that in details are given by $\boldsymbol{q}_{v}=\left(q_{v x}, q_{v y}, q_{v z}\right)^{T} \in \mathbb{R}^{3}$, $q_{v x}, q_{v y}, q_{v z}$ respectively represent the translation of mobile platform in $\mathrm{x}$ - and $\mathrm{y}$-direction and rotation in z-direction, and $\boldsymbol{q}_{r} \in \mathbb{R}^{7}$ represents the joint coordinates of the manipulator. As shown in Fig. 2, $\boldsymbol{T}_{M}^{W}, \boldsymbol{T}_{R}^{M}$ and $\boldsymbol{T}_{E E}^{R}$ represent respectively the transformations from $\Sigma_{M}$ to $\Sigma_{W}$, from $\Sigma_{R}$ to $\Sigma_{M}$ (constant), and from $\Sigma_{E E}$ to its $\Sigma_{R}$

The six dimensional representation of $\Sigma_{E E}$ w.r.t. $\Sigma_{W} \boldsymbol{x}_{w}(\boldsymbol{q})$ can be extracted in angle-axis form, which is used by the robotic arm as follows:

$$
\boldsymbol{x}_{w}(\boldsymbol{q})=\boldsymbol{W}\left(\boldsymbol{x}\left(\boldsymbol{q}_{r}\right)+\boldsymbol{d}_{0}\right)+\boldsymbol{q}_{v}^{\prime}\left(\boldsymbol{q}_{v}\right)
$$

where $\boldsymbol{W}=\left(\begin{array}{cc}\boldsymbol{\operatorname { R o t }}\left(\boldsymbol{z}, q_{v z}\right) & \underset{0}{\mathbf{3} \times \mathbf{3}}\end{array}\right) \in \mathbb{R}^{6 \times 6}, \boldsymbol{\operatorname { R o t }}\left(\boldsymbol{z}, q_{v z}\right)$ represents the rotation matrix around z-axis with the angle $q_{v z}, \boldsymbol{x}\left(\boldsymbol{q}_{r}\right) \in \mathbb{R}^{6}$ represents the six dimensional representation of $\Sigma_{E E}$ w.r.t. $\Sigma_{R}$, $\boldsymbol{d}_{0} \in \mathbb{R}^{6}$ is the deviation between the $\Sigma_{M}$ and $\Sigma_{R}$, and $\boldsymbol{q}_{v}^{\prime}=$ $\left(q_{v x}, q_{v y}, 0,0,0, q_{v z}\right)^{T}$.

MOCA Jacobian matrix w.r.t. $\Sigma_{W}$ can be derived directly from equation (14):

$$
\begin{aligned}
\boldsymbol{J}_{w}(\boldsymbol{q}) & =\frac{\partial \boldsymbol{x}_{w}(\boldsymbol{q})}{\partial \boldsymbol{q}^{T}}=\left(\begin{array}{ll}
\frac{\partial \boldsymbol{x}_{w}(\boldsymbol{q})}{\partial \boldsymbol{q}_{v}^{T}} & \frac{\partial \boldsymbol{x}_{w}(\boldsymbol{q})}{\partial \boldsymbol{q}_{r}^{T}}
\end{array}\right) \\
& =\left(\begin{array}{ll}
\boldsymbol{J}_{m} \quad \boldsymbol{W} \boldsymbol{J}_{r}
\end{array}\right)
\end{aligned}
$$

where $\boldsymbol{J}_{m} \in \mathbb{R}^{6 \times 3}$ represents the contribution of the mobile platform velocity, $\boldsymbol{J}_{r} \in \mathbb{R}^{6 \times 7}$ is the arm Jacobian matrix.

Based on the whole-body forward kinematics $\boldsymbol{x}_{w}(\boldsymbol{q})$, the whole-body Jacobian matrix $\boldsymbol{J}_{w}(\boldsymbol{q})$ and the dynamic model interface provided by the manipulator, the whole-body Cartesian inertial $\boldsymbol{\Lambda}_{w}\left(\boldsymbol{x}_{w}\right)$, the Cartesian Coriolis/centrifugal $\boldsymbol{\mu}\left(\boldsymbol{x}_{w}, \dot{\boldsymbol{x}}_{\boldsymbol{w}}\right)$ and the null-space projection matrix $\boldsymbol{N}(\boldsymbol{q})$ can be computed from equation (8), (9) and (12), respectively.

The desired Cartesian stiffness in (7) is estimated from the human arm as described in Section III, and the damping terms are computed with the Double Diagonalization Design [26].

\section{HUMAN-RoBot LOCO-MANIPULATION INTERFACE}

In this section, a human-robot loco-manipulation interface is developed to enable intuitive control of MOCA mobility and arm interaction. Fig. 3 illustrates the two control modes, i.e., Manipulation and Locomotion.

For the Locomotion mode, MOCA arm and base controllers are decoupled to avoid asynchronized dynamic responses between the manipulator and the mobile platform. ${ }^{1}$ In this phase, the virtual torques of the mobile-base admittance controller are computed based on the estimation of human $\mathrm{CoP}$ resulting from the body inclinations, which correspond to the directions of motion in remote environment.

On the other hand, for the Manipulation mode, the operator's arm position and stiffness commands are tracked in real-time and replicated by the MOCA's whole-body impedance controller. Details of the human-robot interface are explained below.

\footnotetext{
${ }^{1}$ Although this can be avoided using the coupled whole-body controller, however, several hard constraints must be imposed to avoid the motion of arm w.r.t. the mobile base.
} 


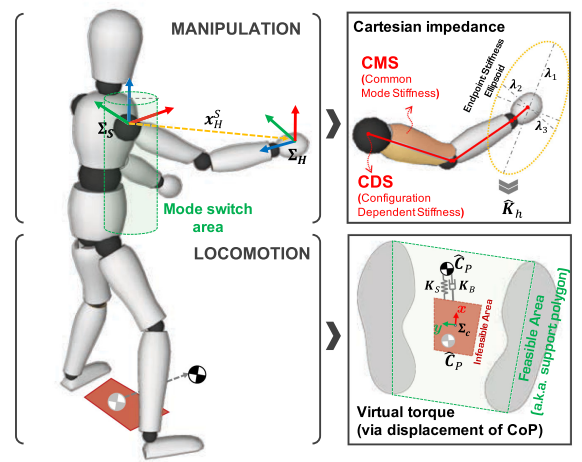

Fig. 3. Whole-body Manipulation (top) is performed through the teleimpedance interface, Locomotion (bottom) is controlled with virtual torques, that are based on the $\mathrm{CoP}$ displacement.

\section{A. Locomotion Mode}

In the Locomotion mode, the motion of the mobile platform is controlled on the basis of the position of the whole-body $\mathrm{CoP}$ of the subject, which is estimated by means of a Statically Equivalent Serial Chain (SESC) technique, as described in [28], using data collected through the 3D motion tracking of the subject. In this phase, the manipulator is controlled independently with a Cartesian impedance controller and no command is sent from human side. In order to make it compliant with possible obstacles, low stiffness parameters are set in the controller.

By taking into account the human's support polygon (see bottom picture in Fig. 3), that is the horizontal region bounded by the top and the bottom of the right and left foot, we defined a "dead-zone" area which corresponds to a specific percentage of the support polygon (by default the $50 \%$, but it can be set differently) and is treated as a no-movements area for the mobile platform. This consideration is to avoid undesired movements of the mobile platform resulted by small, involuntary body sways of the operator.

Accordingly, we computed the displacement of the CoP $\Delta C_{P}$, which is set to zero if the CoP lies inside the sub-polygon, while it is equal to the distance between the CoP and the closest side of the sub-polygon, otherwise. The resulting CoP displacement is then used to compute the virtual torques by

$$
\boldsymbol{\tau}_{v}^{v i r}=\boldsymbol{K}_{S} \Delta \boldsymbol{C}_{P}+\boldsymbol{K}_{D} \Delta \dot{\boldsymbol{C}}_{P}
$$

where $\boldsymbol{K}_{S} \in \mathbb{R}^{2 \times 2}$ and $\boldsymbol{K}_{D} \in \mathbb{R}^{2 \times 2}$ are the virtual stiffness and damping matrices respectively. Here, only translational motion on $x$ and $y$ axes is considered. The computed torque $\tau_{v}^{v i r}$ is sent to MOCA "Admittance Interface" to employ the Locomotion mode.

The virtual stiffness and damping matrices values were experimentally chosen based on the resulting mobile-based velocity, which is achieved by body inclinations of the operator.

\section{B. Whole-Body Manipulation Mode}

Tele-impedance [7] is a control paradigm developed in the last five years. It consists in tele-operating a robot through an impedance controller by measuring and replicating the user's limb pose and impedance on the slave robot in real-time. The user's impedance is estimated by monitoring the muscles' activity through the use of surface electromyography (sEMG) and interpreted to estimate the impedance of the human limb. The estimation may involve a detailed muscle model, usually a Hill-based one or a derivation of it.

Towards the tracking of the human physical interaction behaviour in 3D space using a principled simplification approach, recently [8], the tele-impedance concept is extended based on the dependency of the arm endpoint stiffness to both geometric human arm configuration (Configuration Dependent Stiffness CDS) and muscular activity (Common Mode Stiffness - CMS).

CDS includes the effect of arm configuration and muscle moment arms that contribute to the variations in the geometry of the arm endpoint stiffness. The arm kinematics is retrieved by the $3 \mathrm{D}$ motion tracking system, which enables the computation of the arm Jacobian $\left(\boldsymbol{J}_{a}\left(\boldsymbol{q}_{a}\right)\right)$, with $\boldsymbol{q}_{a} \in \mathbb{R}^{7}$ being the arm joint angles. Using the muscle attachment points, and the length variations over the joint angles, i.e. the muscle Jacobian ( $\left.\boldsymbol{J}_{\text {musc }}\left(\boldsymbol{q}_{a}\right)\right)$ can be computed online [8].

CMS, on the other hand, implements a coordinated coactivation of the arm muscles, while its tracking is achieved by a co-contraction index $\left(a_{c c}\right)$, calculated from the dominant and easily accessible muscles of the arm for surface electromyography measurements, i.e. the Biceps Brachii (BB) and Triceps Brachii (TB). Through a pre-define muscle synergy matrix $\left(\boldsymbol{K}_{\text {syn }}\right)$, this index contributes to modifications in the volume of the endpoint stiffness ellipsoid.

Finally, the congruence conservative transformation from the joint space to the Cartesian space of the human arm can be written to obtain the estimated endpoint stiffness $\hat{\boldsymbol{K}}_{h}$ (see details in [8]):

$$
\hat{\boldsymbol{K}}_{h}=\boldsymbol{J}_{a}^{+T}\left(\boldsymbol{q}_{a}\right)\left(\boldsymbol{J}_{m u s c}^{T}\left(\boldsymbol{q}_{a}\right) a_{c c} \boldsymbol{K}_{s y n} \boldsymbol{J}_{m u s c}\left(\boldsymbol{q}_{a}\right)\right) \boldsymbol{J}_{a}^{+}\left(\boldsymbol{q}_{a}\right) .
$$

The identification of the parameters in (17) was achieved in an off-line experimental phase as described in [8], and will not be repeated here.

\section{Control Architecture}

The components illustrated in the previous sections need to be integrated in a unique framework, in order to build an effective system capable of following the operator's intentions. The control flow of the software architecture is depicted in Fig. 2. The operator, assuming a standing pose, is provided with two ways to command the robot, the Locomotion mode and the Manipulation mode, and is able to switch between them at any time through the "Mode switch" action. This command mode change is enabled by some predefined operator's arm gestures. When the user arms are at his/her sides, the Locomotion mode is activated, while by raising the right arm, the user can switch to Manipulation mode. This motion is detected when the human hand position (i.e., position at the origin of $\boldsymbol{\Sigma}_{H}$ with respect to $\boldsymbol{\Sigma}_{S}$ ) is placed at outside of the cylindrical constraint (see Fig. 3). To switch back to the Locomotion mode, the operator needs first to raise also his/her left arm and then to take down both arms going back to the starting pose with both arms at his/her sides. Note that, simply lowering the right arm to switch mode, without using the left arm, would not be a feasible option, since the robotic arm would still be subject to teleoperation movements. 


\section{A. Locomotion}

In the Locomotion mode, the "Center of Pressure Estimation" module receives as input the 3D motion tracking data and estimates the human whole-body CoP that is given in turn, to the "Virtual Torques Computation" module, whose output are the virtual torques $\tau_{v}^{v i r}$ (see Section III-A) that are sent to the mobile platform "Admittance Interface". The robotic arm is instead controlled by a standard Cartesian impedance control. In this mode, the mobile platform and the robotic arm are controlled independently to avoid unnecessary excessive movements of the upper part of the system and to achieve a smoother behavior.

\section{B. Manipulation}

On the contrary, in the Manipulation mode, the upper and the lower part of the system are commanded by a "Whole-body Impedance Controller" as a unified framework. The "Motion Planner" unit takes as input the human hand displacement w.r.t. its initial pose (with $\boldsymbol{\Sigma}_{H}$ as reference frame) and at every time step adds it to the initial robot pose computed in the world frame $\boldsymbol{\Sigma}_{W}$. The human impedance, estimated by the "Tele-impedance Interface", is directly mapped to the robot Cartesian impedance.

\section{EXPERIMENTS}

To validate the proposed method, we carried out experiments switching between the two modes. The operator teleoperated the robot, localized in a remote environment, relying on the visual data provided by the camera mounted on MOCA. These data were streamed on a screen located in front of him/her. The 3D whole-body motion tracking data were retrieved thanks to a MVN Biomech suit (Xsens Tech) provided with 17 interconnected inertial measurement units (IMU). The frequency of the human-robot loco-manipulation interfaces is based on the 3D motion tracking frequency, i.e. $80 \mathrm{~Hz}$, while the impedance controllers run in real-time at $1 \mathrm{KHz}$, and the "Admittance Interface" at $300 \mathrm{~Hz}$.

The user had to accomplish the following subtasks: using the Locomotion mode, guide the robot in front of a closet, switch to Manipulation mode to open the closet door, change back to the first mode to move forward, and finally switch again to Manipulation mode to grab a drill placed inside the closet and pierce a wall on the left.

Fig. 4 represents the Locomotion mode, in which the user drove the robot in front of the closet. The plots show the operator CoP displacement $\Delta C_{P}$, on which are based the virtual torques $\boldsymbol{\tau}_{v}^{v i r}$ calculated as in (16), where $\boldsymbol{K}_{S}=300 \mathrm{~N} / \mathrm{m}$ and $\boldsymbol{K}_{D}$ is set to the critical damping. $\boldsymbol{\tau}_{v}^{v i r}$ are used as input to the "Admittance Interface" module, that computed the mobile platform desired velocity $\dot{\boldsymbol{q}}^{\text {des }}$ through (5). The last row of the figure describes how the robot end-effector pose changes w.r.t. the world frame $\boldsymbol{\Sigma}_{W}$.

Once the robot was guided close enough to the closet, the operator raised the right arm to switch to Manipulation mode. In this stage, depicted on Fig. 5(a), the user had to open the door of the closet. The first two plots highlight the coupling between the movements of the human hand w.r.t. his/her shoulder $\left(\boldsymbol{x}_{H}^{S}\right)$ and the motion of the robot end-effector w.r.t. the world frame $\left(\boldsymbol{x}_{E E}^{W}\right)$. The third plot depicts the diagonal values of the

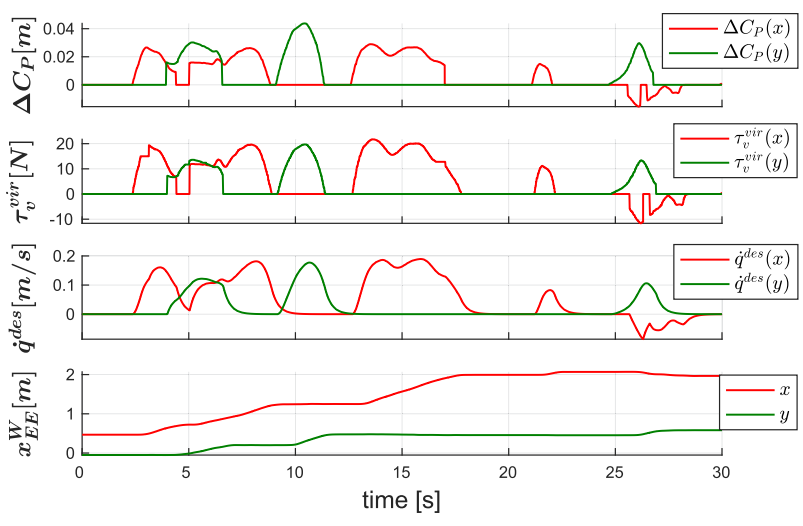

Fig. 4. Locomotion mode: the human operator, by bending frontally and/or laterally, modifies his/her CoP position. Based on its displacement $\Delta \boldsymbol{C}_{P}$, the virtual torques $\boldsymbol{\tau}_{v}^{v i r}$ are computed and the "Admittance Interface" translates them in a desired velocity $\dot{\boldsymbol{q}}^{\text {des }}$ through which MOCA changes its pose $\boldsymbol{X}_{E E}^{W}$.

Cartesian stiffness matrix $\boldsymbol{K}$ estimated by the "Tele-impedance Interface" module and set to the robot "Whole-body Impedance Controller". Since the door opening had to be carried out mainly on the $x$ axis, as shown by the external interaction forces $\boldsymbol{F}_{\text {ext }}$, we can notice that the impedance gains reached high values only on that axis remaining compliant on $y$ and $z$ axes. Only in this way a successful execution of the task was possible, in fact to open the door the robot had to comply with the door constraints especially in $y$ direction while remaining stiff in $x$ direction to be able to open it. This avoided the generation on unnecessary high interaction forces in $y$ and $z$ axes.

After having opened the closet door, the operator switched back to Locomotion mode and moved in a configuration in which it was feasible to both grab the drill inside the closet and pierce a wall on its left. We omit the relative plots since they are very similar to the ones shown in Fig. 4. The plots describing the last Manipulation mode are depicted on Fig. 5(b). In the first part $(t \simeq 8)$ the operator grabbed the drill, as highlighted by the sudden negative variation of the external interaction forces $\boldsymbol{F}_{\text {ext }}$ on $z$ axis given by the tool weight $(2 \mathrm{Kg})$. In this phase, the Cartesian stiffness $\boldsymbol{K}$ gains are high only in $x$ direction since the human arm is fully extended frontally to reach the tool inside the closet. Next, the operator led the robot to the left as it can be noticed by the positive variation on $y$ axis in the first two plots, and pierced multiple times the wall. This time the Cartesian stiffness $\boldsymbol{K}$ values are high only in $y$ direction, as the external interaction forces $\boldsymbol{F}_{\text {ext }}$. Due to the increase of stiffness in $y$ axis, the drill can penetrate inside the wall, and remain relatively compliant in other axes so that any misalignment is gently treated.

To show the adaptability of the framework to different tasks and people, another subject performed a valve turning task. Since the results were similar, the plots are not shown here. However, the experiment is included in the multimedia attachment of the manuscript.

\section{DISCUSSION}

The whole-body impedance controller improves the robot interaction performance in terms of accuracy and safety, in 

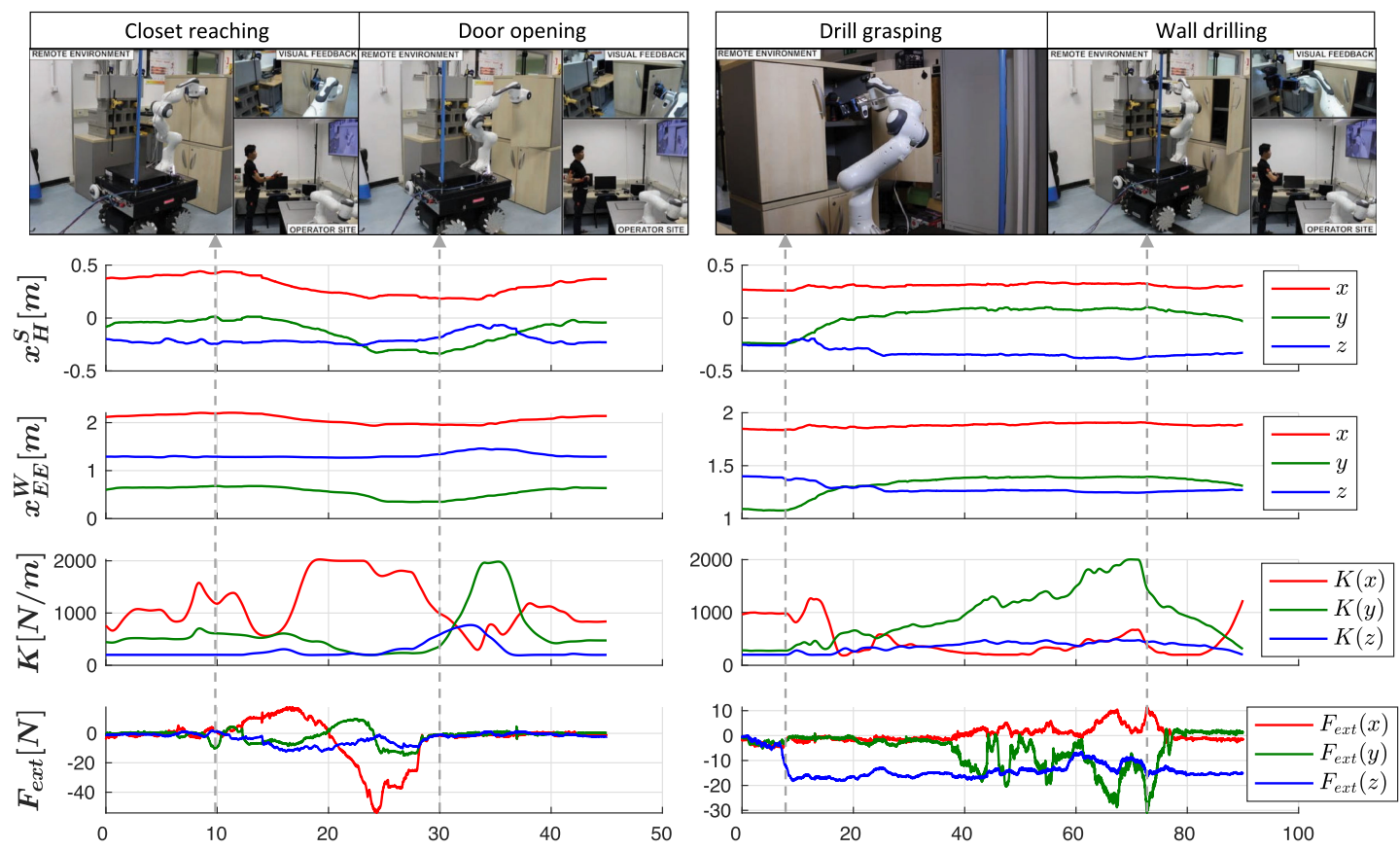

(a)

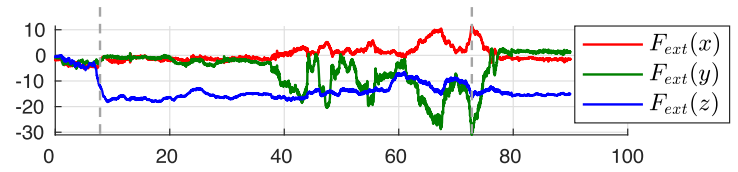

(b)

Fig. 5. Manipulation mode: the human operator performs the door opening (a), and the wall drilling (b). The first two plots show the coupling between the position of the human hand w.r.t. his/her shoulder frame $\boldsymbol{X}_{H}^{S}$, and the robot end-effector pose expressed in the world frame $\boldsymbol{X}_{E E}^{W}$. The third plot depicts the Cartesian stiffness estimated by the "Tele-impedance Interface" that is mapped onto the robot "Whole-body Impedance Controller". In the last plot, the external interaction forces are represented, highlighting the pulling of the closet handle on $\mathrm{x}$-axis (a), and the wall piercing on the $\mathrm{y}$-axis (b). A video of the experiment is available in the multimedia material.

comparison to a decoupled system, i.e. Cartesian impedance control on the robotic arm and admittance control on the mobile platform. A good example is characterized by the door opening phase: the $\mathrm{x}$-axis forces exerted by the door handle while pulling it, make the mobile platform move back on the same axis. Without this coupled control, only the arm would move back and the door would crash against the mobile platform, resulting in a failure of the task and causing a damage to the environment and to the robot itself. The integration of a collision avoidance algorithm would additionally increase the interaction safety, which will be addressed in our future works.

Future developments will also include the extension of the framework to dual-arm manipulation. In this case, the CoP interface brings even more benefits, since the two human arms/hands are dedicated to command the tele-impedance interface. Nevertheless, to highlight the importance and intuitiveness-of-use of the developed CoP interface in this work, we performed additional experiments and compared its performance to a case where robot mobility was controlled using a joystick. The task was to navigate the mobile platform using the two locomotion interfaces (CoP or joystick), and to perform a painting task on a wall in two different areas. The operators were asked to zoom in the camera view manually, using a button, after each painting action to control the quality of the painting tasks. This implied that, when using the joystick, the subjects had to grasp and switch between the joystick and the camera button repeatedly. Ten healthy subjects participated to the experiments.

After the execution of the two tasks, they were asked to fill out a Likert scale questionnaire to compare the CoP and

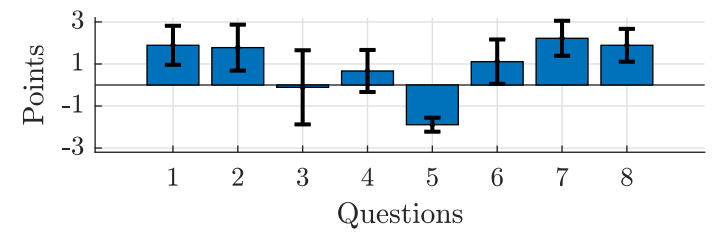

Fig. 6. Likert scale questionnaire scores about the human subjective evaluation comparing the CoP and the joystick interfaces.

the joytick interface. The questionnaire included 8 statements. Q1: Accomplishing the task with the CoP interface was less mentally demanding; Q2: Coordination of the robot mobility and manipulation was more intuitive using the CoP interface. Q3: Accomplishing the task with the Joystick interface was more physically demanding. Q4: I had to work harder to accomplish my level of performances using the Joystick interface. Q5/Q6: I felt more discouraged, stressed, and annoyed using the CoP/joystick interface. Q7: The CoP interface has a higher potential to make the execution of complex remote loco-manipulation tasks easier for a user. Q8: Overall, I felt I was improving the mixed loco-manipulation performance with the CoP interface. The possible answers ranged from strongly disagree to strongly agree, with an assigned score of -3 and +3 , respectively. The results, illustrated in Fig. 6 , show that the users found the presented interface more intuitive, less mentally demanding, and with a higher potential to make the execution of complex loco-manipulation tasks easier, in comparison with a joystick interface. 
We also measured the average time to complete the task with the two modes obtaining $117,7 \mathrm{~s}$ for the CoP interface and $158,9 \mathrm{~s}$ using the joystick, across all subjects and the experiments.

\section{CONCLUSION}

In this work, we introduced our new mobile manipulator MOCA (MObile Collaborative robotic Assistant). We also proposed a novel framework for whole-body teleoperation, including the human-robot loco-manipulation interfaces to command it, in which the operator was provided with two modes to control the robotic platform. In the Locomotion mode, the user was able to move the mobile platform by simply bending in the direction of the desired movement exploiting the displacement of his/her center of pressure, and the robotic arm was controlled with a Cartesian impedance controller. Switching to the Manipulation mode, the operator could teleoperate MOCA by moving his/her hand through a tele-impedance controller, which retrieved the human pose and impedance parameters that served as input to the whole-body impedance controller. We experimentally validated our framework in a challenging remote interaction scenario to illustrate its high potential. The intuitiveness-of-use of the proposed CoP-based locomotion interface was also compared to a traditional joystick, revealing it's higher coordination performance.

\section{ACKNOWLEDGMENT}

The authors would like to thank Dr. Cheng Fang for his contributions to the muscle Jacobian module. The experiments were approved by the ethics committee Azienda Sanitaria Locale Genovese (ASL) N.3 (Protocollo IIT_HRII_001 (rif. interno:108/2018)).

\section{REFERENCES}

[1] W. Bluethmann et al., "Robonaut: A robot designed to work with humans in space," Auton. Robots, vol. 14, nos. 2/3, pp. 179-197, 2003.

[2] T. Klamt et al., "Supervised autonomous locomotion and manipulation for disaster response with a centaur-like robot," in Proc. IEEE/RSJ Int. Conf. Intell. Robots Syst., 2018, pp. 1-8.

[3] J. Leven et al., "Davinci canvas: A telerobotic surgical system with integrated, robot-assisted, laparoscopic ultrasound capability," in Proc. Int. Conf. Med. Image Comput. Comput.-Assisted Intervention, 2005, pp. 811-818.

[4] F. Espinosa, M. Salazar, D. Pizarro, and F. Valdés, "Electronics proposal for telerobotics operation of P3-DX units, " in, Remote and Telerobotics. Rijeka, Croatia: InTech, 2010.

[5] A. M. Okamura, "Methods for haptic feedback in teleoperated robotassisted surgery," Ind. Robot: An Int. J., vol. 31, no. 6, pp. 499-508, 2004.

[6] M. Tufail and C. W. de Silva, "Impedance control schemes for bilateral teleoperation," in Proc. 9th Int. Conf. Comput. Sci. Educ., 2014, pp. 44-49.

[7] A. Ajoudani, N. G. Tsagarakis, and A. Bicchi, "Tele-impedance: Towards transferring human impedance regulation skills to robots," in Proc. IEEE Int. Conf. Robot. Autom., 2012, pp. 382-388.
[8] A. Ajoudani, C. Fang, N. Tsagarakis, and A. Bicchi, "Reduced-complexity representation of the human arm active endpoint stiffness for supervisory control of remote manipulation," Int. J. Robot. Res., vol. 37, no. 1, pp. 155-167, 2018.

[9] E. Hocaoglu and V. Patoglu, "Tele-impedance control of a variable stiffness prosthetic hand," in Proc. IEEE Int. Conf. Robot. Biomimetics, 2012, pp. 1576-1582.

[10] M. Laghi et al., "Shared-autonomy control for intuitive bimanual telemanipulation," in Proc. IEEE-RAS 18th Int. Conf. Humanoid Robots, 2018 .

[11] N. Tsagarakis et al., "Walk-man: A high-performance humanoid platform for realistic environments," J. Field Robot., vol. 34, pp. 1225-1259, 2017.

[12] J. Englsberger et al., "Overview of the torque-controlled humanoid robot TORO," in Proc. 14th IEEE-RAS Int. Conf. Humanoid Robots, 2014, pp. 916-923.

[13] C. Borst et al., "Rollin' Justin-mobile platform with variable base," in Proc. IEEE Int. Conf. Robot. Autom., 2009, pp. 1597-1598.

[14] H. Iwata and S. Sugano, "Design of human symbiotic robot TWENDYONE," in Proc. IEEE Int. Conf. Robot. Autom., 2009, pp. 580-586.

[15] A. Spada, M. Cognetti, and A. De Luca, "Locomotion and telepresence in virtual and real worlds," in Human Friendly Robotics. New York, NY, USA: Springer, 2019, pp. 85-98.

[16] L. Peneo et al., "Robust real-time whole-body motion retargeting from human to humanoid," in Proc. IEEE-RAS 18th Int. Conf. Humanoid Robots, 2018, pp. 425-432.

[17] J. D. Will, K. L. Moore, and I. K. Lynn, "Optimizing human-robot teleoperation interfaces for mobile manipulators," Ind. Robot: Int. J., vol. 40, no. 2, pp. 173-184, 2013.

[18] A. Hentout, M. Benbouali, I. Akli, B. Bouzouia, and L. Melkou, "A telerobotic human/robot interface for mobile manipulators: A study of human operator performance," in Proc. Int. Conf. Control, Decis. Inf. Technol., 2013, pp. 641-646.

[19] V. H. Andaluz et al., "Transparency of a bilateral tele-operation scheme of a mobile manipulator robot," in Proc. Int. Conf. Augmented Reality, Virtual Reality Comput. Graph., 2016, pp. 228-245.

[20] J. Rebelo, T. Sednaoui, E. B. den Exter, T. Krueger, and A. Schiele, "Bilateral robot teleoperation: A wearable arm exoskeleton featuring an intuitive user interface," IEEE Robot. Autom. Mag., vol. 21, no. 4, pp. 62-69, Dec. 2014.

[21] T. Hulin et al., "The DLR bimanual haptic device with optimized workspace," in Proc. IEEE Int. Conf. Robot. Autom., 2011, pp. 3441-3442.

[22] M. G. Catalano, G. Grioli, E. Farnioli, A. Serio, C. Piazza, and A. Bicchi, "Adaptive synergies for the design and control of the Pisa/IIT SoftHand," Int. J. Robot. Res., vol. 33, no. 5, pp. 768-782, 2014.

[23] A. Dietrich et al., "Whole-body impedance control of wheeled mobile manipulators," Auton. Robots, vol. 40, no. 3, pp. 505-517, 2016.

[24] Y. Yamamoto, "Control and coordination of locomotion and manipulation of a wheeled mobile manipulators," Ph.D. dissertation, Univ. Pennsylvania, Philadelphia, PA, USA, 1994.

[25] X. Yun and Y. Yamamoto, "Internal dynamics of a wheeled mobile robot," in Proc. IEEE/RSJ Int. Conf. Intell. Robots Syst., 1993, vol. 2, pp. 1288-1294.

[26] C. Ott, Cartesian Impedance Control of Redundant and Flexible-Joint Robots, vol. 49. New York, NY, USA: Springer, 2008.

[27] O. Khatib, "A unified approach for motion and force control of robot manipulators: The operational space formulation," IEEE J. Robot. Autom., vol. RA-3, no. 1, pp. 43-53, Feb. 1987.

[28] W. Kim, J. Lee, N. Tsagarakis, and A. Ajoudani, "A real-time and reduced-complexity approach to the detection and monitoring of static joint overloading in humans," in Proc. Int. Conf. Rehabil. Robot., 2017, pp. 828-834. 